\title{
Reviews
}

\section{Asymptomatic bacteriuria in pregnancy}

\author{
Jennifer Perera $^{1}$ \\ Sri Lanka Journal of Obstetrics and Gynaecology 2009; 31: 108-109
}

Key words: Asymptomatic bacteriuria, pregnancy, infection.

\section{Introduction}

Urinary tract infection (UTI) is common during pregnancy. It could be either symptomatic or asymptomatic which complicates the diagnostic process. It has been reported that $6 \%$ of pregnant women have asymptomatic bacteriuria ${ }^{1}$. Untreated asymptomatic bacteriuria is associated with adverse maternal and perinatal morbidity and mortality.

Significant bacteriuria may exist in asymptomatic patients. Significant bacteriuria is traditionally defined as $>10^{5}$ colony forming units or bacteria/ml of urine as at this level a chance of contamination from distal urethra is less than $1 \%$.

At concentrations of $10^{3}-10^{4}$ bacteria/ml, there is a $50 \%$ chance that contamination is responsible ${ }^{2}$ for a positive culture and in such instances most laboratories would request a repeat testing with a midstream sample of urine. Isolation of the same organism in the second sample is more indicative of significant bacteriuria

\section{Pathogenesis}

The relatively acidic $\mathrm{pH}$, high osmolality and high urea concentration in urine is generally bacteriostatic to most bacteria. Furthermore, in an anatomically normal urinary tract, infection is prevented by unobstructed antegrade flow of urine.

The physiological changes, both hormonal and mechanical that occur in the urogenital tract during pregnancy, increases the potential for colonization by pathogenic bacteria. During pregnancy the bladder volume increases and detrusor tone decreases. Additionally, progesterone relaxes ureteric smooth muscle causing dilatation of ureters which is further aggravated due to pressure from the expanding uterus.

\footnotetext{
${ }^{1}$ Professor of Microbiology, Department of Microbiology, Faculty of Medicine, University of Colombo.

E-mail: jennifer_perera55@yahoo.com
}

All these factors lead to urinary stasis, dysfunctional ureteric valves and vesicoureteric reflux which facilitates bacterial colonization and ascending infection. Seventy percent of pregnant women develop glycosuria and this in combination with the physiological lowering of urine osmolality will further facilitate bacterial colonization ${ }^{3}$.

Maternal immunity is modified during pregnancy to favour implantation and development of the embryo. Research suggests that the immune response is modulated from a predominantly cell mediated response to a predominantly humoral immune response ${ }^{4}$. Humoral responses are less efficient as cell surface major histocompatibility receptors are not necessarily recognized during the response. Therefore this may result in less efficient recognition of bacterial cell surface proteins which prevents removal of bacteria facilitating colonization and infection by bacteria. Although the effect of immune system status during pregnancy in infectious pathology remains controversial, its role in facilitating infection by uropathogens cannot be disregarded.

Diabetes mellitus, gestational diabetes and prepregnancy UTI are other risk factors that predispose to UTI during pregnancy. Finally it is important to understand that prevalence of UTI increases with age and in low socioeconomic groups ${ }^{2}$ and medical interventions such as catheterization predisposes to ascending bacteriuria.

\section{Bacteriology}

The organisms that cause UTI during pregnancy are the same as those found in non pregnant patients. Escherichia coli accounts for $80-90 \%$ of infections ${ }^{5}$. Other Gram negative rods such as Proteus mirabilis and Klebsiella pneumoniae can also be cultured. Gram positive cocci such as Staphylococcus saprophyticus, other coagulase negative staphylococci and group B streptococci are less common causes of UTI, although infections by group B streptococci have important implications for management of pregnancy. Less common organisms that may cause UTI include enterococci, Gardenerella vaginalis, Chlamydia spp, Ureaplasma spp and Candida albicans. Although vaginal colonisation with Group B streptococci is strongly 
associated with preterm rupture of membranes, labour and delivery and causes neonatal sepsis, evidence relating group B streptococcal bacteriuria with similar consequences is less well established ${ }^{6}$.

\section{Clinical significance}

Asymptomatic significant bacteriuria is associated with adverse maternal outcomes. These include symptomatic cystitis in approximately $30 \%$ of patients, development of pyelonephritis (in up to $50 \%$ ) and preterm labour and delivery. Pyelonephritis in the pregnant patient leads to septicaemia in 10 $20 \%$ of cases and acute respiratory distress syndrome in $2 \%$. Associated adverse foetal outcomes include prematurity, low birth weight and increased perinatal mortality ${ }^{7}$. In addition there are increased maternal risks reported for pre-eclampsia, anaemia, chorioamnionitis and post partum endometritis in patients with significant bacteriuria. Fetal risks include fetal growth retardation, stillbirth, mental retardation and development delay. It is postulated that direct bacterial endotoxin damage is responsible.

\section{Management}

A Cochrane review ${ }^{8}$ established that, treatment of significant bacteriuria reduces the risk of pyelonephritis in pregnancy and consequently, the risks of preterm delivery and low birth weight. The American College of Obstetrics and Gynaecology recommends that a urine culture be done at the first antenatal visit. Cost benefit analysis shows that screening is cost effective with a bacteriuria prevalence of $>2 \%$ in the antenatal population. Rapid testing reagent strips for screening for asymptomatic bacteriuria show lower sensitivity (50\%) when compared to traditional culture hence not recommended 9 .

Treatment should be directed by the culture and sensitivity testing as antibiotic sensitivity pattern of the implicated organisms varies markedly from one population to another. Additionally it is important to use an antibiotic with an established safety profile. The appropriate antibiotic should be continued for at least 7 days as a review of short courses of antimicrobials found insufficient evidence to confirm treatment efficacy with shorter schedules of therapy ${ }^{10}$.

It is important to do a 'test of cure' urine culture 12 weeks after completion of antimicrobial therapy. If the 'test of cure' culture is positive a repeat course of antibiotics is recommended based on urine culture and sensitivity report and suppressive therapy ( eg Nitrofurantoin $50 \mathrm{mg} /$ day) is recommended after a negative 'test of cure' urine culture. It is recommended that patients with two or more episodes of bacteriuria are followed up with monthly repeat cultures until delivery, to ensure urine sterility ${ }^{11}$.

\section{Key points:}

- Asymptomatic significant bacteriuria in pregnancy is associated with significant maternal and perinatal mortality.

- All women should be screened for asymptomatic bacteriuria during early pregnancy

- The gold standard for detecting bacteriuria is by culture of a midstream specimen of urine.

- Antibiotic therapy should be continued for 7 days as shorter courses are less effective during pregnancy.

- A 'test of cure' urine culture after completion of treatment is required to ensure response to therapy.

- Two or more episodes of significant bacteriuria warrant suppressive therapy

\section{References}

1. Kass EH. Maternal urinary tract infection. New York State Journal of Medicine 1962; 2822-6.

2. McCormick T, Ashe RG, Kearney PM. Urinary tract infection in pregnancy. The Obstetrician and Gynaecologist 2008; 10: 156-62.

3. Asscher AW, Sussman M, Waters WE, Davis RH, Chick S. Urine as a medium for bacterial growth. The Lancet 1966: 2: 1037-41.

4. Wegmann TG, Lin H, Guilbert L, Mosmann TR. Bidirectional cytokine interactions in the maternal-foetal relationship: is successful pregnancy a $\mathrm{TH} 2$ phenomenon? Immunology Today 1993; 14: 353- 6.

5. Polk BF. Urinary tract infection in pregnancy. Clinical Obstetrics and Gynaecology 1979; 22: 285-92.

6. Moller M, Thomsen AC, Borch K, Dinesen K, Zdravkovic M. Rupture of foetal membranes and premature delivery associated with group B streptococci in urine of pregnant women. The Lancet 1984; 2: 69 - 70.

7. Mittendorf R, Williams MA, Kass EH. Prevention of preterm delivery and low birth weight associated with asymptomatic bacteriuria. Clinical Infectious Disease 1992; 14: 927-32.

8. Smaill F. Antibiotics for asymptomatic bacteriuria in pregnancy. Cochrane Database of Systematic Reviews 2001; 2: CD000491.

9. National Collaborating Centre for Women's and Children's Health. Antenatal care. Routine Care for Healthy Pregnant Women. London. RCOG Press 2003.

10. Harris RE, Gilstrap LC, Pretty A. Single dose antimicrobial therapy for asymptomatic bacteriuria in pregnancy. Obstetrics and Gynaecology 1982; 59: 546-9.

11. Chen KT. UTI in pregnancy: 6 questions to guide therapy. OBG Management 2004; 16(11): 36-54. 